\title{
NOTE ON A FAMILY OF VOLTERRA EQUATIONS
}

\author{
KENNETH B. HANNSGEN 1
}

ABSTRACT. We prove that the solutions of a certain family of Volterra integrodifferential equations are uniformly bounded. We use this result to determine the asymptotic behavior of the solution of a Volterra equation in Hilbert space.

1. Statement of results. Let $u(t, \lambda)$ denote the solution of

$$
u^{\prime}(t, \lambda)+\lambda \int_{0}^{t} a(t-s) u(s, \lambda) d s=0, \quad u(0, \lambda)=1
$$

(primes denote differentiation with respect to the first variable).

Recently D. F. Shea and S. Wainger [9] proved that

$$
\int_{0}^{\infty}|u(t, \lambda)| d t<\infty
$$

if $\lambda>0$ and if $a(t)$ satisfies the conditions

$$
a(t) \text { is nonnegative, nonincreasing, and convex on }(0, \infty) \text {, }
$$

$$
\int_{0}^{1} a(t) d t<\infty \text {, and } a(t) \not \equiv a(\infty),
$$

$$
\lambda a^{*}(\zeta) \equiv \int_{0}^{\infty} e^{-\zeta t} \lambda a(t) d t \neq-\zeta \quad(\operatorname{Re} \zeta \geq 0)
$$

Using a simple identity based on the resolvent formula for (1.1), we prove the following estimate.

Theorem 1. If (1.3) holds and $\lambda_{0}>0$, then

$$
|w(t, \lambda)| \leq 4 \int_{0}^{\infty}\left|u\left(t, \lambda_{0}\right)\right| d t \quad\left(\lambda_{0} \leq \lambda<\infty, 0 \leq t<\infty\right),
$$

where $w(t, \lambda)=\int_{0}^{t} u(s, \lambda) d s$.

This extends the results in [5], where we required (among other things) that $a(t)$ be twice differentiable if $a \notin L^{1}(0, \infty)$. The extension is significant because it takes in the special case where

Received by the editors June 29, 1973 and, in revised form, October 9, 1973. AMS (MOS) subject classifications (1970). Primary 45D05.

Key words and phrases. Asymptotic behavior, convex, Hilbert space, integrodifferential equations, selfadjoint linear operator, viscoelasticity, Volterra equations.

${ }^{1}$ Partially supported by NSF Grant GP-27973. 
$a(t)$ is piecewise linear with changes of slope only at integral multiples of $t=t_{0}>0$.

As we showed in [4],

$$
u(t, \lambda) \rightarrow 0 \text { and } \lambda w(t, \lambda) \rightarrow A \equiv\left(\int_{0}^{\infty} a(t) d t\right)^{-1} \geq 0 \quad(t \rightarrow \infty)
$$

if (1.3) holds, unless (1.6) holds and

$$
\lambda=\lambda_{j} \equiv j^{2}\left[4 \pi^{2} / a(0) t_{0}^{2}\right] \quad(j=1,2, \cdots) .
$$

In the latter case, we set

$$
\begin{aligned}
\gamma & =3-[a(\infty) / a(0)], \quad \omega_{j}=\left(\lambda_{j} a(0)\right)^{1 / 2}=2 \pi j / t_{0}, \\
u_{j}(t) & =2 \gamma^{-1} \cos \omega_{j} t, \quad w_{j}(t)=2\left[\gamma \omega_{j}\right]^{-1} \sin \omega_{j} t+A / \lambda .
\end{aligned}
$$

Then (1.6) and (1.8) imply

$$
u\left(t, \lambda_{j}\right)-u_{j}(t) \rightarrow 0, \quad w\left(t, \lambda_{j}\right)-w_{j}(t) \rightarrow 0 \quad(t \rightarrow \infty) .
$$

For reference we note that when (1.3) holds

$$
|u(t, \lambda)| \leq \sqrt{2} \quad(0<\lambda<\infty, 0 \leq t<\infty),
$$

but that $\sup _{0<\lambda, 0 \leq t}|w(t, \lambda)|=\infty$. See [5] for the proofs of these facts. We also remark that (1.4) holds whenever (1.3) holds, except when (1.6) and (1.8) hold [4, Lemma 5]. On the other hand, Shea and Wainger proved (1.2) under conditions weaker than those stated above.

We may use Theorem 1 to study the Volterra equation

$$
y(t)+\mathbf{L} \int_{0}^{t} h(t-s) y(s) d s=\xi+t \kappa,
$$

where $\mathbf{L}$ is a selfadjoint linear operator defined on the dense subspace $\mathfrak{D}$ of the Hilbert space $\mathcal{H}, \mathrm{L} \geq \lambda_{0} I\left(\lambda_{0}>0\right)$, and $h(t)=\int_{0}^{t} a(s) d s$. Here $\xi$ and $\kappa$ are prescribed elements of $\mathcal{H}$. As in [6], we may use (1.5) and (1.10) to deduce the representation

$$
y(t)=\int_{\lambda_{0}}^{\infty} d \mathbf{E}_{\lambda}[u(t, \lambda) \xi+w(t, \lambda) \kappa]
$$

( $\left\{\mathbf{E}_{\boldsymbol{\lambda}}\right\}$ is the spectral family corresponding to $\mathbf{L}, \mathbf{E}_{\lambda}=\mathbf{E}_{\lambda_{-}-}$) and determine the asymptotic behavior of $y(t)$.

Remark. For fixed $t, u(t, \lambda)$ and $w(t, \lambda)$ are continuous functions of $\lambda$, even at $\lambda=\lambda_{j}$ when (1.6) holds. This is shown by an elementary argument (similar to that for a linear ordinary differential equation with a regular linear parameter) using (1.1) and the easily checked equation

$$
w^{\prime}(t, \lambda)+\lambda \int_{0}^{t} a(t-s) w(s, \lambda) d s=1 .
$$


This justifies our use of integrals like (1.12).

Theorem 2. Suppose (1.3) holds and let

$$
\Omega(t)=A \mathbf{L}^{-1} \kappa+\sum_{j=1}^{\infty} \mathbf{F}_{j}\left[u_{j}(t) \xi+w_{j}(t)_{\kappa}\right],
$$

where $\mathbf{F}_{j}=\mathbf{E}_{\lambda^{+}}-\mathbf{E}_{\lambda}, \lambda=\lambda_{j}$, and the sum is omitted unless (1.6) holds. Then

$$
\lim _{t \rightarrow \infty}\|y(t)-\Omega(t)\|=0
$$

Theorem 2 was proved in [6] with smoothness restrictions excluding (1.6) and hence without the sum in $\Omega(t)$. Other recent studies of equations like (1.11) with hypotheses similar to ours include those of C. M. Dafermos [1] and of R. C. MacCamy and J. S. W. Wong [8]. Dafermos discusses applications to viscoelasticity. A. Friedman and M. Shinbrot [3] and later Friedman [2] used spectral decomposition methods to study more general classes of equations in Banach spaces.

Recently [7] we proved a restricted analogue of Theorem 1 for (1.1) with $\lambda a(t)$ replaced by $a(t, \lambda)$; this result also had implications for certain equations in Hilbert space.

2. Proof of Theorem 1. Let $\lambda \geq \lambda_{0}$. Using Laplace transforms or otherwise, one verifies that

$$
u(t, \lambda)=u\left(t, \lambda_{0}\right)+\frac{\lambda-\lambda_{0}}{\lambda} \int_{0}^{t} u^{\prime}(t-s, \lambda) u\left(s, \lambda_{0}\right) d s .
$$

Therefore,

$$
w(t, \lambda)=w\left(t, \lambda_{0}\right)+\frac{\lambda-\lambda_{0}}{\lambda} \int_{0}^{t}[u(t-s, \lambda)-1] u\left(s, \lambda_{0}\right) d s .
$$

Since $0<\left(\lambda-\lambda_{0}\right) / \lambda<1,(1.10)$ and (2.1) yield (1.5).

3. Proof of Theorem 2. If (1.6) does not hold, the proof is the same as that given in [6] so we assume here that (1.6) holds. To shorten the formulas we also assume $A=0$. Without loss of generality we take $\lambda_{0}<\lambda_{1}$. Then (1.2) holds with $\lambda=\lambda_{0}$.

Using (1.1), (1.13), (1.5), and (1.10), one sees by direct substitution that (1.12) gives the unique continuous solution of (1.11). (See [6] for details of this argument.)

We shall also need the following continuity result from [6]. (See "Remarks on piecewise linear kernels.' ) 
Theorem A. If (1.3) and (1.6) hold, $u(t, \lambda)$ and $w(t, \lambda)$ are continuous in $\lambda$, uniformly in $0 \leq t<\infty$, except at $\lambda=\lambda_{j}(j=1,2,3, \ldots)$, where the cont $i$ nuity is not uniform.

By (1.5) and (1.10), B $=\sup _{t \geq 0, \lambda \geq \lambda_{0}}(|u(t, \lambda)|+|w(t, \lambda)|)<\infty$. Let $\epsilon>0$, and choose a positive integer $J$ and a positive number $\delta$ such that

$$
\int_{\Delta}\left[d\left(\mathbf{E}_{\lambda} \xi, \xi\right)+d\left(\mathbf{E}_{\lambda} \kappa, \kappa\right)\right]<\epsilon,
$$

where

$$
\Delta=\bigcup_{j=1}^{J}\left(\left[\lambda_{j}-\delta, \lambda_{j}\right] \cup\left[\lambda_{j}+, \lambda_{j}+\delta\right]\right) \cup\left[\lambda_{J}+\delta, \infty\right),
$$

and $\delta$ is taken small enough to make $\lambda_{j}-\delta>\lambda_{j-1}+\delta(j=1,2, \ldots)$. Next set $\Delta_{1}=\left[\lambda_{0}, \lambda_{1}-\delta\right], \Delta_{j}=\left[\lambda_{j-1}+\delta, \lambda_{j}-\delta\right](j=2,3, \ldots, J)$. Then

$$
y(t)-\Omega(t)=\int_{\Delta} d \mathbf{E}_{\lambda}\left[u(t, \lambda) \xi+w(t, \lambda)_{\kappa}\right]
$$

$$
\begin{aligned}
& +\sum_{j=1}^{J} \mathbf{F}_{j}\left\{\left[u\left(t, \lambda_{j}\right)-u_{j}(t)\right] \xi+\left[w\left(t, \lambda_{j}\right)-w_{j}(t)\right]_{\kappa}\right\} \\
& +\sum_{j=1}^{J} \int_{\Delta_{j}} d \mathbf{E}_{\lambda}\left[u(t, \lambda) \xi+w(t, \lambda)_{\kappa}\right]-\sum_{j=J+1}^{\infty} \mathbf{F}_{j}\left[u_{j}(t) \xi+w_{j}(t)_{\kappa}\right] .
\end{aligned}
$$

By (1.7), (1.9), and Theorem $A$, there is a $T>0$ such that $t \geq T$ implies

$$
\left|u\left(t, \lambda_{j}\right)-u_{j}(t)\right|+\left|w\left(t, \lambda_{j}\right)-w_{j}(t)\right|<\epsilon \quad(j=1,2, \cdots:, J),
$$

and $|u(t, \lambda)|+|w(t, \lambda)|<\epsilon\left(\lambda \in \Delta_{1} \cup \ldots \cup \Delta_{J}\right)$.

Then the two finite sums in (3.3) are bounded in norm by $2 \epsilon(\|\xi\|+\|\kappa\|)$ $(t \geq T)$. By (3.1) the other two terms in (3.3) are bounded in norm by $2 \epsilon\left[B+\gamma^{-1}\left(1+\omega_{1}^{-1}\right)\right]$. Since $\epsilon$ was arbitrary, this establishes (1.14).

\section{REFERENCES}

1. C. M. Dafermos, An abstract Volterra equation with applications to linear viscoelasticity, J. Differential Equations 7(1970), 554-569. MR 41 \#305.

2. A. Friedman, Monotonicity of solutions of Volterra integral equations in Banach space, Trans. Amer. Math. Soc. 138(1969), 129-148. MR 39 \#3359.

3. A. Friedman and M. Shinbrot, Volterra integral equations in Banach space, Trans. Amer. Math. Soc. 126(1967), 131-179. MR 34 \#6571.

4. K. B. Hannsgen, Indirect abelian theorems and a linear Volterra equation, Trans. Amer. Math. Soc. 142(1969), 539-555. MR $39 \# 7364$.

5. $22-30$. 
6. K. B. Hannsgen, A Volterra equation in Hilbert space, SIAM J. Math. Anal. (to appear).

7. - A linear Volterra equation in Hilbert space, SIAM J. Math. Anal. (to appear).

8. R. C. MacCamy and J. S. W. Wong, Stability theorems for some functional equations, Trans. Amer. Math. Soc. 164(1972), 1-37. MR 45 \#2432.

9. D. F. Shea and S. Wainger, Variants of the Wiener-Lévy theorem, with applications to stability problems for some Volterra integral equations, Amer. J. Math. (to appear).

DEPARTMENT OF MATHEMATICS, VIRGINIA POLYTECHNIC INSTITUTE AND STATE UNIVERSITY, BLACKSBURG, VIRGINIA 24061 